\title{
66. THE MAJOR PLANETS AS POWERFUL TRANSFORMERS OF COMETARY ORBITS
}

\author{
E. I. KAZIMIRCHAK-POLONSKAYA \\ Institute for Theoretical Astronomy, Leningrad, U.S.S.R.
}

\begin{abstract}
The equations of motion of many of the comets of the Jupiter and Saturn families have been integrated for a period of $400 \mathrm{yr}$. Several examples illustrate the role of the major planets, especially Jupiter, in the evolution of these orbits: in such cases comets may be captured by Jupiter from Saturn's family or ejected from Jupiter's family into Saturn's, and so on; the possible escape of a comet along a hyperbolic orbit and the capture by Jupiter of a comet from a hyperbolic orbit are also demonstrated. Fictitious comets of the Uranus and Neptune families are also studied. It is concluded that a combination of diffusion theory and capture theory is in good agreement with the observations and makes it possible to consider both long- and short-period comets as part of a unified cometary complex.
\end{abstract}

\section{Introduction}

The research presented here is concerned with the evolution of cometary orbits inside the planetary system for a period of several centuries and with the determination of the roles of the giant planets (Jupiter, Saturn, Uranus, and Neptune) as powerful transformers in that evolution.

On account of the great amount of information available we are obliged to restrict ourselves here to discussing only a few important problems. They may be formulated as follows:

(1) Determination of the most interesting features of the great transformations and of the general evolution of cometary orbits.

(2) Elucidation of the influence of close approaches to Jupiter on the subsequent discovery of comets.

(3) Illustration of the agreement of deduction from classical capture theory with the results of our studies.

(4) Demonstration of the absence of contradictions between the observations and deductions from the capture theory.

(5) Presentation of the main stages in the evolution of cometary orbits by combining diffusion theory with capture theory.

First of all, to avoid possible misunderstandings that sometimes arise in discussions of our work (Vsekhsvyatskij, 1969, pp. 278-280; Sitarski, 1968), we shall elucidate the difference between 'numerical theories of cometary motion' and 'investigations of the evolution of cometary orbits'. The difference is very important as it concerns both the object and method of investigation.

In creating a theory of the motion of a comet the principal aim is to obtain the best conceivable agreement with observations over large intervals of time. Therefore, when the theory is being developed, all perceptible planetary perturbations and nongravitational effects should be taken into account with high precision. The agreement has to be to seconds and even fractions of seconds of arc. 
It is different when we are studying the long-term evolution of cometary orbits: here the investigator is interested in the general process of orbital transformation and in the main factors affecting this process. It is sufficient to work to a precision of decimals of degree and even whole degrees.

When developing the theory of the motion of a comet the investigator is interested only in the period covered by the observations, in the course of which it is very rare for a comet to pass through Jupiter's sphere of action while the effects of passages through the spheres of action of the other planets cannot be detected at all. Very close approaches to Jupiter cause great difficulties, and when they do occur we must take special pains to avoid a gap in the theory during the time of close approach.

When studying the evolution of cometary orbits, however, the particular interest lies precisely in these approaches to the major planets, for one of the main purposes of these investigations is the establishment of the roles played by the major planets in the evolution of cometary orbits, and this role can be detected most obviously when a comet passes through the spheres of action of the planets. It thus becomes inevitable to extend the interval of time over which the study is made to a few centuries or even longer.

We have studied the evolution of the orbits of the short-period comets of different planetary families for an interval of $400 \mathrm{yr}$ (1660-2060) and have ascertained that all the comets of Jupiter's family experience close or moderate approaches to Jupiter; in some cases there have been up to ten approaches during the 400 -yr interval. The comets of Saturn's family approach both Jupiter and Saturn.

We did not discover any close approaches to Uranus and Neptune for the comets belonging to the families of these planets, and the period of $400 \mathrm{yr}$ is too short for fully investigating whether such approaches really occur. Therefore, alongside the real comets we have introduced fictitious comets of the Uranus and Neptune families and have examined the transformations of their orbits in the spheres of action of those planets.

In studying the problem of the evolution of cometary orbits, we may apply either statistical methods - investigating fictitious objects on a large scale; or numerical methods - integrating the actual equations of motion of real and fictitious objects.

\section{Real Comets in the Spheres of Action of Jupiter and Saturn}

We are interested in modelling the true motions of comets as closely as possible. We have therefore conducted this investigation by our new method (KazimirchakPolonskaya, 1972), using our complex of double-precision programmes that minimize the error both in the initial data and in the integration. A heliocentric method was used throughout, and we took into account all perceptible planetary perturbations, and in the case of $\mathrm{P} / \mathrm{Wolf}$ nongravitational effects too.

Starting from the very precise, continuous theory of the motion of $\mathbf{P} /$ Wolf for the whole period of observations, 1884-1967 (Kamieński, 1959; Kazimirchak-Polonskaya, 1972), we have integrated the motion of this comet back from 1884 to 1661 . Initially, we allowed for the perturbations by Venus to Pluto and the nongravitational effects. 
The nongravitational coefficients were varied according to the perihelion distance (Kamieński, 1961), which changed after each approach to Jupiter. We then repeated the calculation excluding the nongravitational effects. The resulting elements for an osculation epoch in 1661 are compared in Table I. It may be noted that the action of

\section{TABLE I}

Comparison of two integrations of $\mathrm{P} /$ Wolf over the interval 1884-1661, with and without nongravitational effects

\begin{tabular}{lccc}
\hline & $\begin{array}{l}\text { With } \\
\text { nongravitational } \\
\text { effects }\end{array}$ & $\begin{array}{l}\text { Without } \\
\text { nongravitational } \\
\text { effects }\end{array}$ & $\begin{array}{l}\text { Difference } \\
\text { with-without }\end{array}$ \\
\hline$M$ & 0.54 & $359: 99$ & +0.55 \\
$\omega$ & 158.12 & 158.15 & -0.03 \\
$\Omega$ & 218.62 & 218.48 & +0.14 \\
$i$ & 25.30 & 25.35 & -0.05 \\
$e$ & 0.4549 & 0.4538 & +0.0011 \\
$a$ (AU) & 4.000 & 4.001 & -0.001 \\
$P$ (yr) & 7.999 & 8.004 & -0.005 \\
$q$ (AU) & 2.180 & 2.185 & -0.005 \\
\hline
\end{tabular}

The elements are referred to the mean equinox 1950.0 and correspond to the epoch 1661 Aug. 20.5 Greenwich Mean Time.

the nongravitational forces does not affect the general course of the evolution of $\mathrm{P} /$ Wolf, even though in the 223-yr interval the comet made two close and two moderate approaches to Jupiter (see Table II).

TABLE II

Approaches of $\mathrm{P} /$ Wolf to Jupiter $\left(\Delta_{\min }<1.000 \mathrm{AU}\right)$

\begin{tabular}{lrlll}
\hline$T_{\min }$ & $\Delta_{\min }$ (AU) & \multicolumn{1}{l}{$T_{\min }$} & $\Delta_{\min }$ (AU) \\
\hline 1757 Jan. 15.4 & 0.465 & 1922 Sept. 27.1 & 0.125 \\
1816 Jan. 4.0 & 0.898 & 2005 Aug. 14.2 & 0.542 \\
1839 Nov. 30.3 & 0.250 & 2041 Mar. 10.1 & 0.600 \\
1875 June 8.5 & 0.116 & & \\
\hline
\end{tabular}

In 1965-1966 N. A. Belyaev and the author completed the investigation of the orbital evolution of 45 short-period comets over 1660-2060. Belyaev studied the orbital evolution of ten comets of the Jupiter family, while the author worked on 35 comets belonging to the families of Jupiter, Saturn, Uranus, and Neptune. Up to the present time we have published the results for 22 typical comets, for which the best initial elements were available (Belyaev, 1966, 1967; Kazimirchak-Polonskaya, 1967a, 1967b, 1968). During the 400 -yr interval those 22 comets made 156 approaches to Jupiter, 35 to Saturn, and 2 to Uranus; among them there were more than 40 passages through Jupiter's sphere of action and one passage through Saturn's. 
Let us consider the main features of the orbital evolution of the most interesting short-period comets and the influence of Jupiter's perturbations on the discovery of these comets.

Figure 1 shows the orbits of the Earth, Jupiter, and five different averaged orbits of $\mathrm{P} /$ Wolf. We may note that this comet

(1) consistently remains in the Jupiter family, that planet securely maintaining the comet's aphelion $(A)$ near its own orbit;

(2) revolves around the Sun along a 'pulsating ellipse' varying with an irregular periodicity depending on the character of the approaches to Jupiter; this feature was discovered by Kamieński (1959).

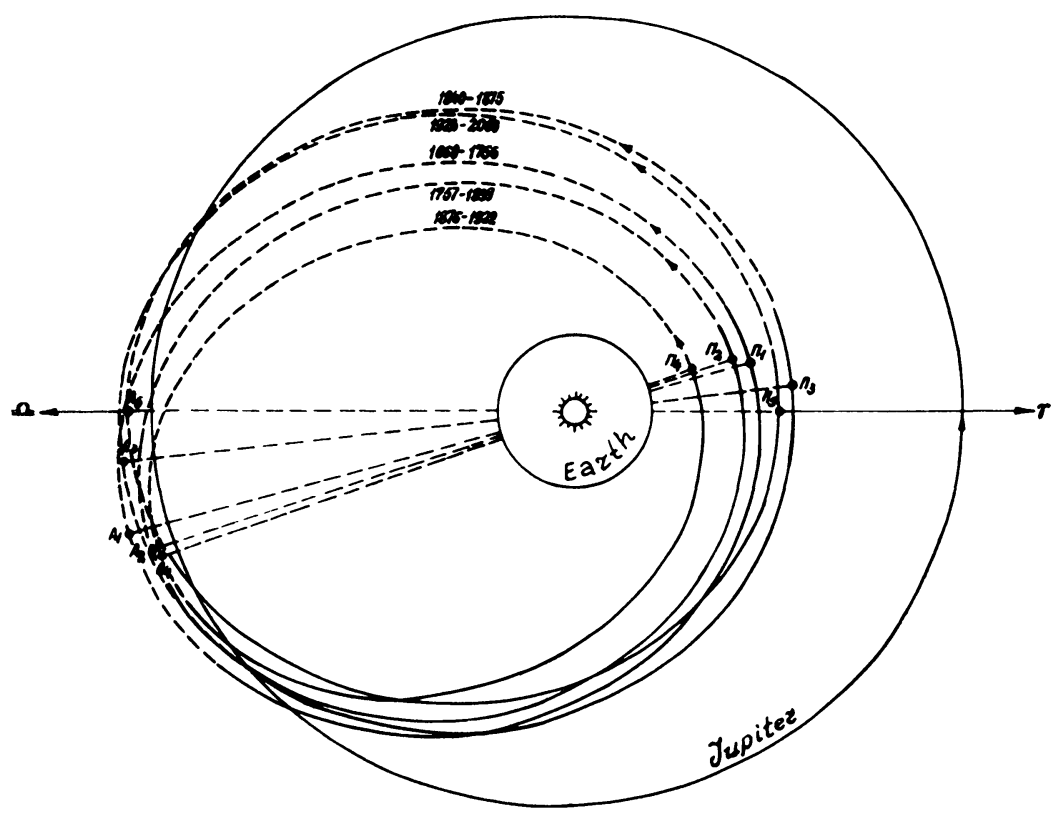

Fig. 1. The orbit of $\mathrm{P} / \mathrm{Wolf}$.

The figure demonstrates graphically that for more than $200 \mathrm{yr}$ the comet was inaccessible to observation on account of the large perihelion distance $q$. After passing through Jupiter's sphere of action in 1875, $q$ was considerably reduced (from 2.4 to $1.6 \mathrm{AU})$, and consequently it became possible for the comet to pass near the Earth. In fact, on 1884 October 1, a month and a half before perihelion, the comet approached the Earth for the first time to a distance of $0.803 \mathrm{AU}$. The increased apparent brightness enabled the comet to be discovered (on 1884 September 17 in Heidelberg) as an object of the ninth magnitude.

An interesting, but different, type of orbital evolution is represented by $\mathrm{P} /$ WolfHarrington, which also remains a member of the Jupiter family for the 400-yr interval. 


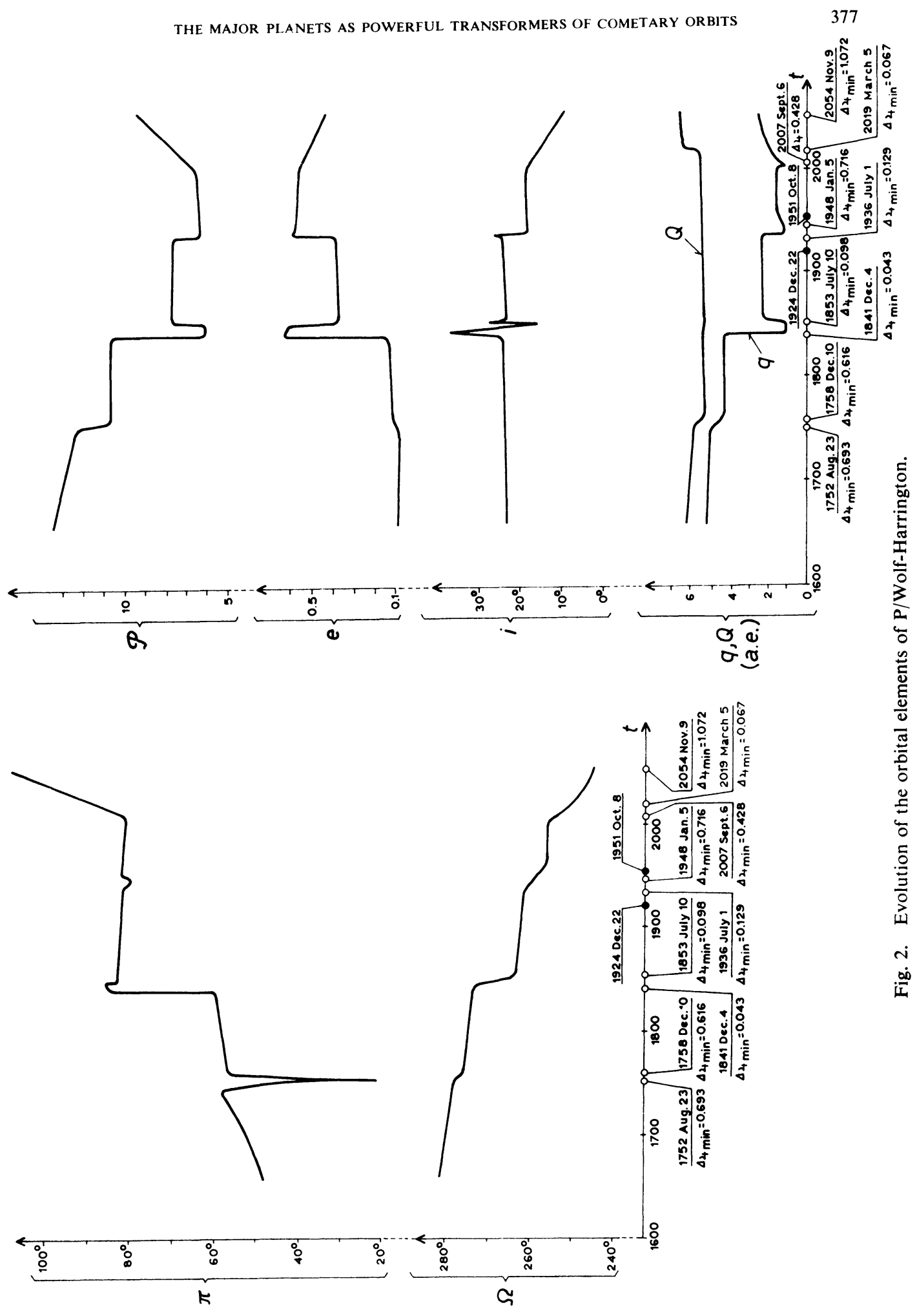


It makes nine approaches to Jupiter, four of them very deeply into its sphere of action (see Table III).

TABLE III

Approaches of $\mathbf{P} /$ Wolf-Harrington to Jupiter

\begin{tabular}{ll}
\hline$T_{\min }$ & $\Delta_{\min }(\mathrm{AU})$ \\
\hline 1841 Dec. & 0.043 \\
1853 July & 0.098 \\
1936 July & 0.129 \\
2019 March & 0.067 \\
\hline
\end{tabular}

Figure 2 demonstrates the changes in the orbital elements of this comet during 1660 2060, while Figure 3 illustrates the actual orbit over 1760-2000, taking into account

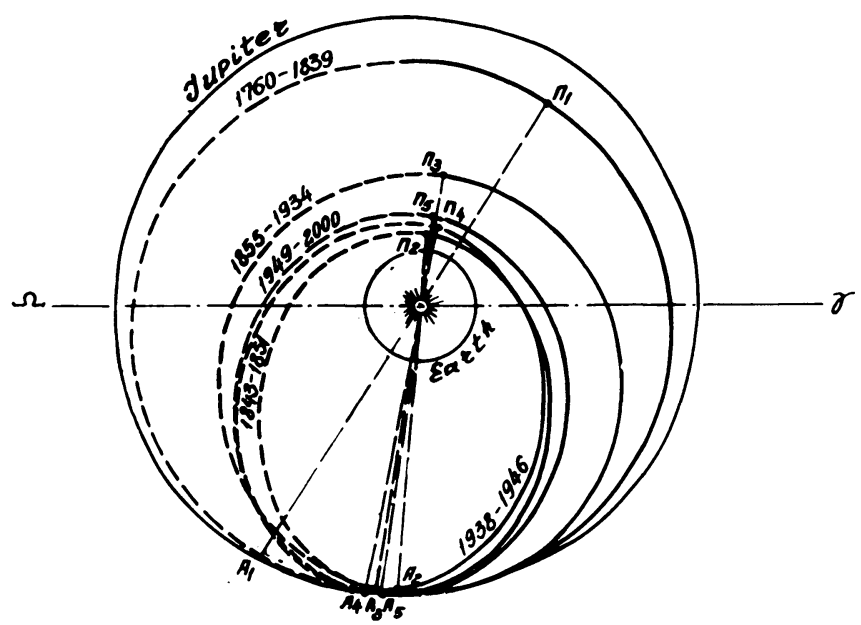

Fig. 3. The orbit of $\mathrm{P} /$ Wolf-Harrington.

the perturbations by Venus to Uranus and starting from the elements by Wiśniewski (1960). The dates on the abscissa of Figure 2 show the nine approaches to Jupiter and the two independent discoveries of the comet (in 1924 and 1951). The more deeply the comet penetrates Jupiter's activity sphere, the more violent are the changes in the orbital elements. The 1841 encounter with Jupiter transformed the orbit from almost a circle (eccentricity $e=0.10$ and period of revolution $P=10.7 \mathrm{yr}$ ) into an eccentric ellipse $(e=0.60, P=6.0 \mathrm{yr})$ while the encounter in 1853 decreased the eccentricity again $(e=0.37, P=7.6 \mathrm{yr})$. The comet was extremely faint in 1924 and not observed well enough for its orbit to be reliably determined; fortunately, the approach to Jupiter in 1936 significantly reduced $q$ (from 2.4 to $1.6 \mathrm{AU}$ ) and made accidental rediscovery of the comet possible in 1951 . 
We shall discuss now another type of comet, one which in the past belonged to the Saturn family and was later captured by Jupiter into its family.

Figure 4 illustrates a series of orbits of $\mathrm{P} / \mathrm{Whipple}$, these being derived on the basis of Dinwoodie's (1960) elements, taking into account the perturbations by Venus to Neptune. From 1660 to 1770 this comet travelled in an orbit far removed from the

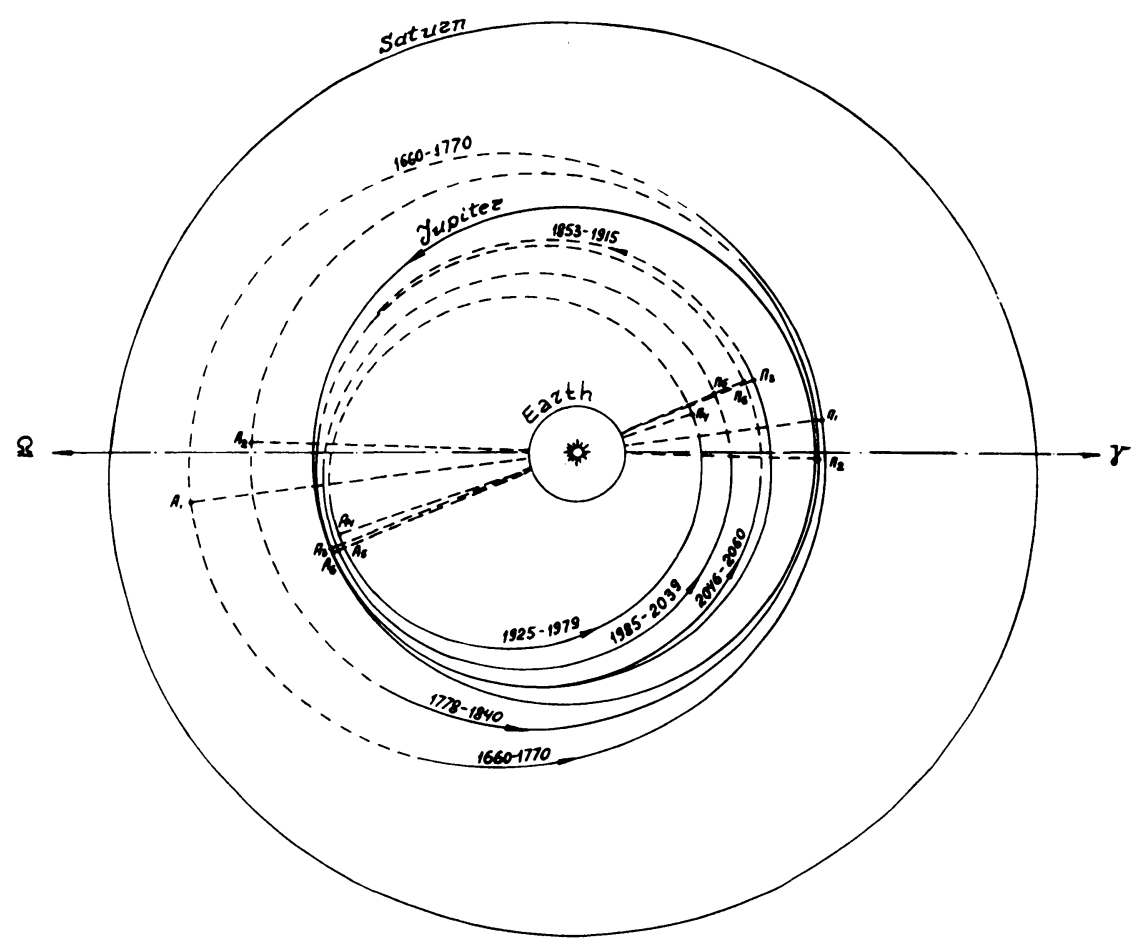

Fig. 4. The orbit of $\mathrm{P} /$ Whipple.

Sun, and since its aphelion $A_{1}$ was located nearer the orbit of Saturn than that of Jupiter it was a member of the Saturn family. Its perihelion $\Pi_{1}$ was in the vicinity of Jupiter's orbit ( $q=5.2 \mathrm{AU}$ ), allowing the possibility of close approaches to Jupiter. From 1770 to 1778 such an approach occurred (lasting 8 years on account of the almost circular form of the cometary orbit), and afterwards the comet moved on a rather different orbit. The second approach to Jupiter was even more characteristic, with planet and comet remaining within a mutual distance of less than $1 \mathrm{AU}$ from 1840 to 1853 . The comet's subsequent orbit was situated completely inside that of Jupiter. That planet had securely captured P/Whipple from the Saturn family. From 1915 to 1925 the comet was near Jupiter again, passing deeply into the sphere of action in 1922, and the resulting perturbations caused the perihelion distance to be decreased quite substantially, rendering discovery of the comet possible in 1933. Further encounters with Jupiter will take place during 1979-1985 and 2039-2046. 
Capture from the Saturn family into the Jupiter family also occurred in the cases of P/Comas Solá (Belyaev, 1967) and P/Shajn-Schaldach. P/Schwassmann-Wachmann 1 was also captured in this manner, about the year 1730 .

An exceptionally interesting type is represented by $\mathrm{P} /$ Oterma. We have investigated the evolution of this orbit, starting from the elements by Herget and Marsden (1961), taking into account the perturbations by Venus to Uranus. During the 400 -yr interval there are four approaches to Jupiter (two of them very deeply into the sphere of action) and one to Saturn. The effects of the two closest encounters are of overriding importance, however, and Figure 5 shows only three averaged orbits for the comet. From 1660 to $1937 \mathrm{P} /$ Oterma had an orbit with perihelion $\Pi_{1}$ slightly outside that of Jupiter

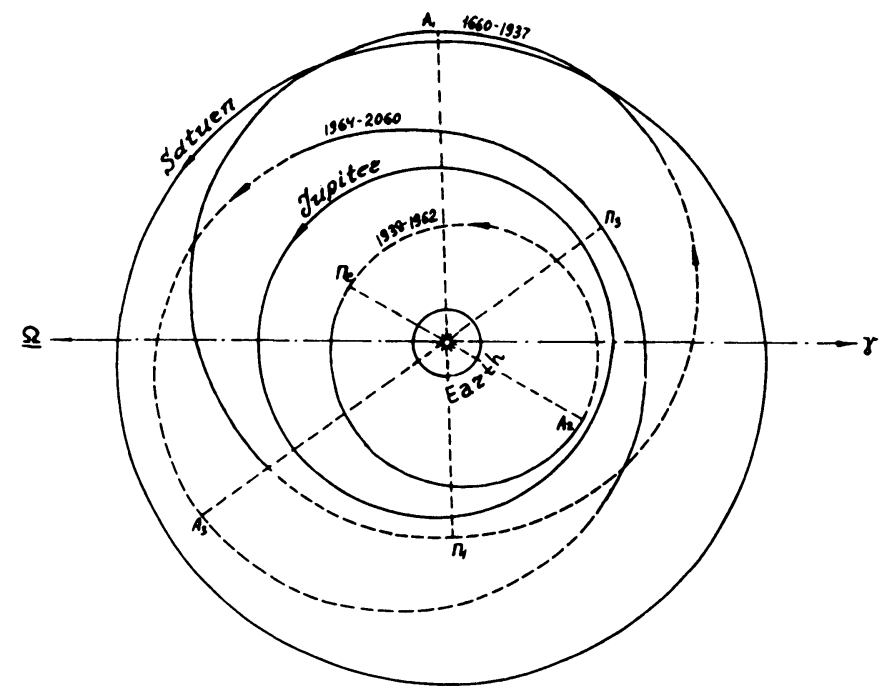

Fig. 5. The orbit of P/Oterma.

and aphelion $A_{1}$ beyond the orbit of Saturn; the revolution period exceeded $20 \mathrm{yr}$. At the end of 1936 the comet entered Jupiter's activity sphere and remained there for more than $2 \mathrm{yr}$, penetrating to $\Delta_{\min }=0.165 \mathrm{AU}$. When the comet left the sphere its orbit was completely different: it was entirely between the orbits of Mars and Jupiter, with its aphelion $A_{2}$ on the inner side of Jupiter's orbit, and its perihelion distance down to $3.4 \mathrm{AU}$ - resulting in the comet's discovery in 1943 already as a member of the Jupiter family. After three revolutions in its new 8-yr orbit it made an even closer approach to Jupiter in $1963\left(\Delta_{\min }=0.095 \mathrm{AU}\right)$ and was subsequently re-ejected into the Saturn family with its perihelion distance again up to 5.4 AU. Moderate approaches to Saturn (in 2008-2016) and to Jupiter (in 2023-2026) will cause further increases in the perihelion distance. During the complete 400-yr interval Jupiter causes the comet's line of apsides to advance $548^{\circ}$ - more than one and a half revolutions.

Turning our attention now to the comets that remain in the Saturn family, we discuss briefly the orbits of $\mathrm{P} /$ Neujmin 3 and $\mathrm{P} / \mathrm{Gale}$. The former comet has nine 
approaches to Jupiter (that in 1850 well into its sphere of action) and six to Saturn, four of which have quite appreciable effects. On this comet the perturbations by Saturn are of the same order as those by Jupiter (e.g. several tens of degrees in $\omega$ and $\Omega) . \mathrm{P} / \mathrm{Gale}$ is noteworthy in that during the $400 \mathrm{yr}$ it experiences the greatest number of approaches: nine to Jupiter and eight to Saturn. In 1798 this comet passed through Saturn's sphere of activity (to $\Delta_{\mathrm{min}}=0.17 \mathrm{AU}$ ), although the actual circumstances of the approach did not produce any great changes in the orbital elements.



Fig. 6. Perturbations in the longitude of the ascending node of a variant orbit of $P / G a l e$ in Saturn's sphere of action.

Since we were interested in the use of comets for revealing the quite unstudied perturbing effects of Saturn within its sphere of activity we varied the conditions of this approach of $\mathrm{P} / \mathrm{Gale}$ and established that the perturbations by Saturn could be as large as those by Jupiter. Figure 6 illustrates the perturbations in the longitude of the ascending node of this fictitious orbit.

In the problem of the evolution of cometary orbits $\mathrm{P} / \mathrm{Neujmin} 1$ deserves particular attention. Although its aphelion is well beyond the orbit of Saturn, this comet belongs to the Saturn family. Our investigations have shown that between 1660 and 2060 there are no approaches to Jupiter at all, although there are six approaches to Saturn, those in 1892 and 1980 to minimum distances of 0.80 and $0.84 \mathrm{AU}$, respectively. The 
evolution of the orbit of this comet is thus determined to a considerable extent by the influence of Saturn.

Of much greater interest is $\mathrm{P} /$ Brooks 2 , which in the past possessed an orbit resembling the present orbit of $\mathrm{P} /$ Neujmin 1. Proceeding from the theory of motion of this comet developed by Dubyago $(1950,1956)$ for the interval $1883-1960$, we extended the calculations to the full 400-yr interval, taking into account the perturbations by Venus to Neptune but without considering the nongravitational effects on this comet. Figure 7 very strikingly illustrates Jupiter's capture of P/Brooks 2 from a situation between the Saturn and Uranus families. According to Dubyago this comet approached

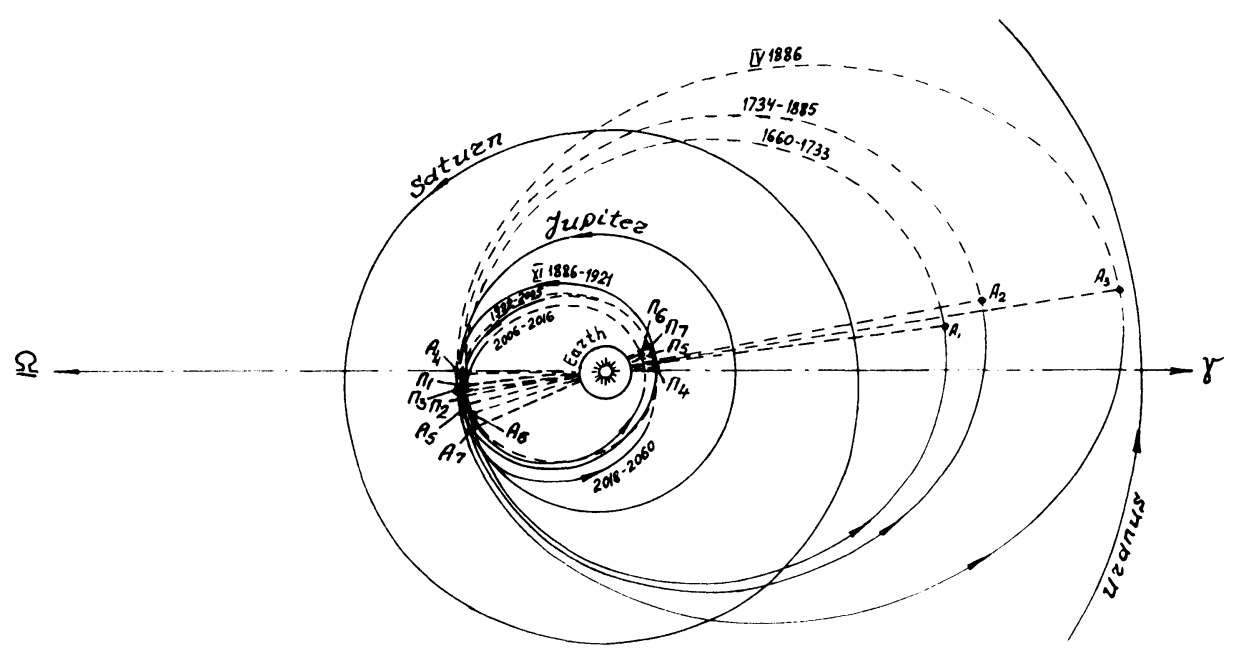

Fig. 7. The orbit of $\mathbf{P} /$ Brooks 2.

Jupiter to within $0.00096 \mathrm{AU}$ in 1886 , and a really catastrophic transformation of the cometary orbit occurred: the ascending and descending nodes changed places, and the perihelion of the former orbit became the aphelion of the new; the perihelion distance decreased from 5.45 to $1.95 \mathrm{AU}$, and it was on account of this that the comet could be discovered in 1889 . The revolution period, initially more than $40 \mathrm{yr}$, decreased to $7 \mathrm{yr}$. A further close approach to Jupiter occurred in 1922, and there will also be approaches in 2005-2006 and in 2017, but the comet nevertheless will remain in the Jupiter family at least until 2060.

The orbital evolution of $\mathrm{P} /$ Lexell is of exceptional interest. First discussed by Lexell $(1777,1778,1781)$, the motion of this comet was subsequently treated in great detail but with allowance only for the perturbations by Jupiter - by Leverrier $(1844,1857)$. Starting from Leverrier's (1844) elements for the 1770 apparition we have studied the evolution of the orbit of $\mathrm{P} /$ Lexell over 1660-2060, considering the perturbations by Venus to Pluto; see Figure 8. Until $1766 \mathrm{P} /$ Lexell had a nearly circular orbit with $q=3 \mathrm{AU}, P=10 \mathrm{yr}$ and was inaccessible to observation. Its close encounter with 
Jupiter in $1767\left(\Delta_{\min }=0.020 \mathrm{AU}\right)$ transformed the orbit into an elongated ellipse with $q=0.67 \mathrm{AU}, P=5.6 \mathrm{yr}$, after which there was an exceptionally close approach to the Earth in 1770 (to within $0.015 \mathrm{AU}$ ), at which time the comet was discovered. After nine years it encountered Jupiter for the second time, on this occasion passing within $0.0015 \mathrm{AU}$ of that planet. The minimum distance was rather similar to that $\mathrm{P} / \mathrm{P}$ Brooks 2 in 1886, although the actual situations were appreciably different: $\mathrm{P} /$ Brooks 2 was near perihelion and approaching the Sun, while $P /$ Lexell was near aphelion and receding; P/Brooks 2 was captured into Jupiter's family, while $\mathrm{P} /$ Lexell was ejected

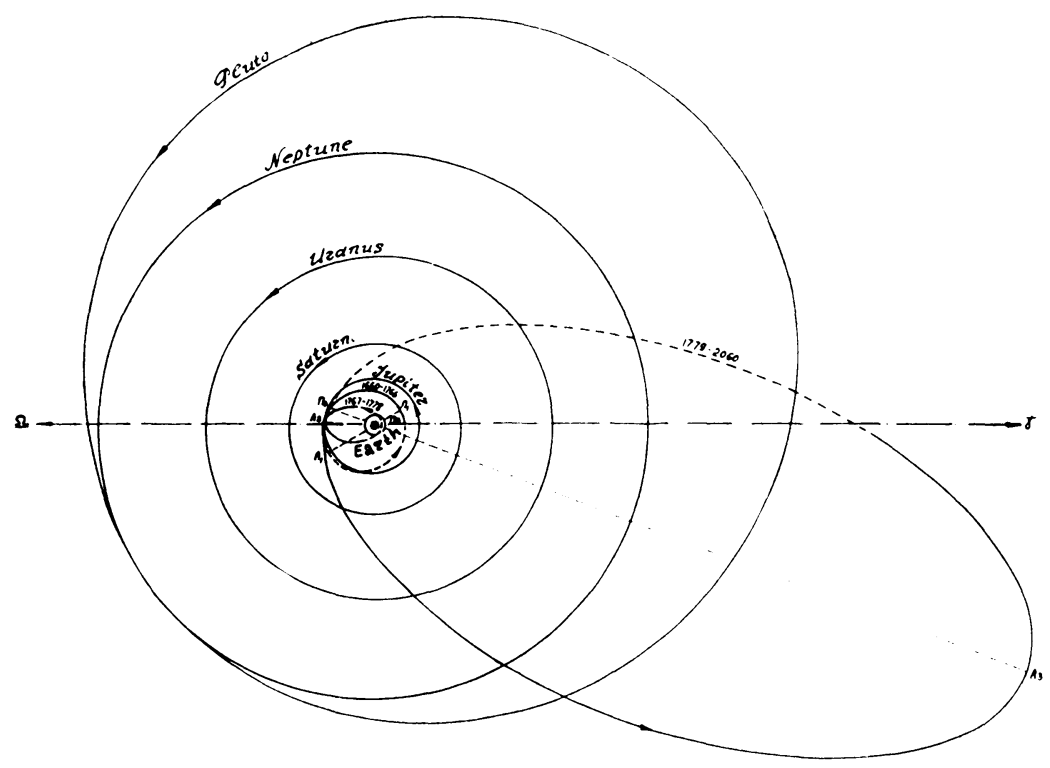

Fig. 8. The orbit of $\mathbf{P} /$ Lexell.

from Jupiter's family into a transplutonian orbit with a revolution period of about $260 \mathrm{yr}$. The perihelion of this new orbit was close to Jupiter's orbit, not far from the aphelion of the former orbit, so $\mathrm{P} /$ Lexell again became unobservable.

We have compared our results on $\mathrm{P} /$ Lexell with those of Leverrier, Schulhof, and Callandreau. Following Leverrier's (1857) example, we varied the initial system of elements and derived several different possible evolutions of the orbit. As before, we confirmed Leverrier's results closely, finding that after the encounter with Jupiter in 1779 some of the solutions gave even more eccentric orbits, a few of them even being hyperbolic. Figure 9 shows one such ellipse and one hyperbola. All the solutions have their perihelia near Jupiter's orbit, confirming that the comet would definitely have been unobservable afterwards. Similar results were also obtained by Callandreau (1892, p. 49).

In connection with the case of $\mathrm{P} /$ Lexell the question naturally arises as to whether 
short-period comets exist that were captured in the past from interstellar space by Jupiter or some other major planet. If there is no real comet, is it possible to invent a fictitious comet with orbital elements similar to those of a real comet, and which would reveal the mechanism of such a capture?

The discovery of $\mathrm{P} /$ Kearns-Kwee in 1963 provided the answer to this interesting question. We investigated the orbital evolution of this comet in 1965, starting from the improved system of elements by Marsden (1964), based on observations over an interval of six months. The equations of motion were integrated forward to 2060 and back

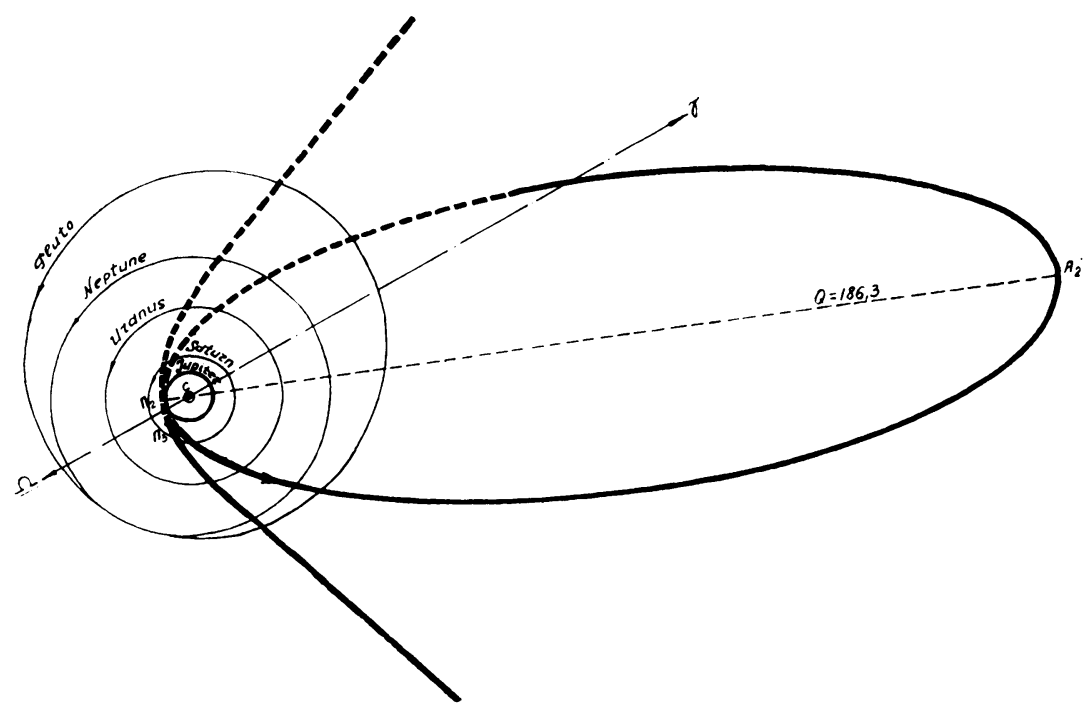

Fig. 9. The possible escape of $\mathrm{P} /$ Lexell on a hyperbolic orbit.

to 1855 , with allowance for the perturbations by Venus to Pluto. The results proved to be exceptionally interesting, showing for the first time the capture by Jupiter of a comet from a hyperbolic orbit. The original comet, moving in a hyperbola, passed within $0.042 \mathrm{AU}$ of Jupiter in 1855 in the vicinity of its perihelion. The great perturbations by Jupiter transformed that orbit into an elongated ellipse whose aphelion (see Figure 10) was initially near the orbit of Neptune. Later, the aphelion $A_{3}$ moved to the boundary of the Neptune and Uranus families. In $1961 \mathrm{P} / \mathrm{Kearns}-\mathrm{Kwee}$ approached Jupiter to within $0.032 \mathrm{AU}$, and that planet captured the comet into its own family, reducing $q$ from 4.3 to $2.2 \mathrm{AU}$ and enabling the comet to be discovered in 1963. There was simultaneously a rotation of the line of apsides by about $90^{\circ}$, and the revolution period decreased from $54 \mathrm{yr}$ to $9 \mathrm{yr}$. With the help of later observations Marsden and Aksnes (1967) determined a more precise orbit and confirmed the enormous transformation of the comet's orbit in 1961. However, neither they nor Sitarski (1968) agreed with the capture of the comet from a hyperbolic orbit in 1855 . This is naturally explained by differences in the initial elements for 1963 and by the increased discrepancies among the elements (especially the mean anomaly and mean motion) before the 
approach to Jupiter in 1961. However, no matter what the conclusions of other researchers may be, the nature of the problem is not changed, and the results obtained are still of significance for the problem of the evolution of cometary orbits. It is important that such a remarkable real object has been observed, and although the

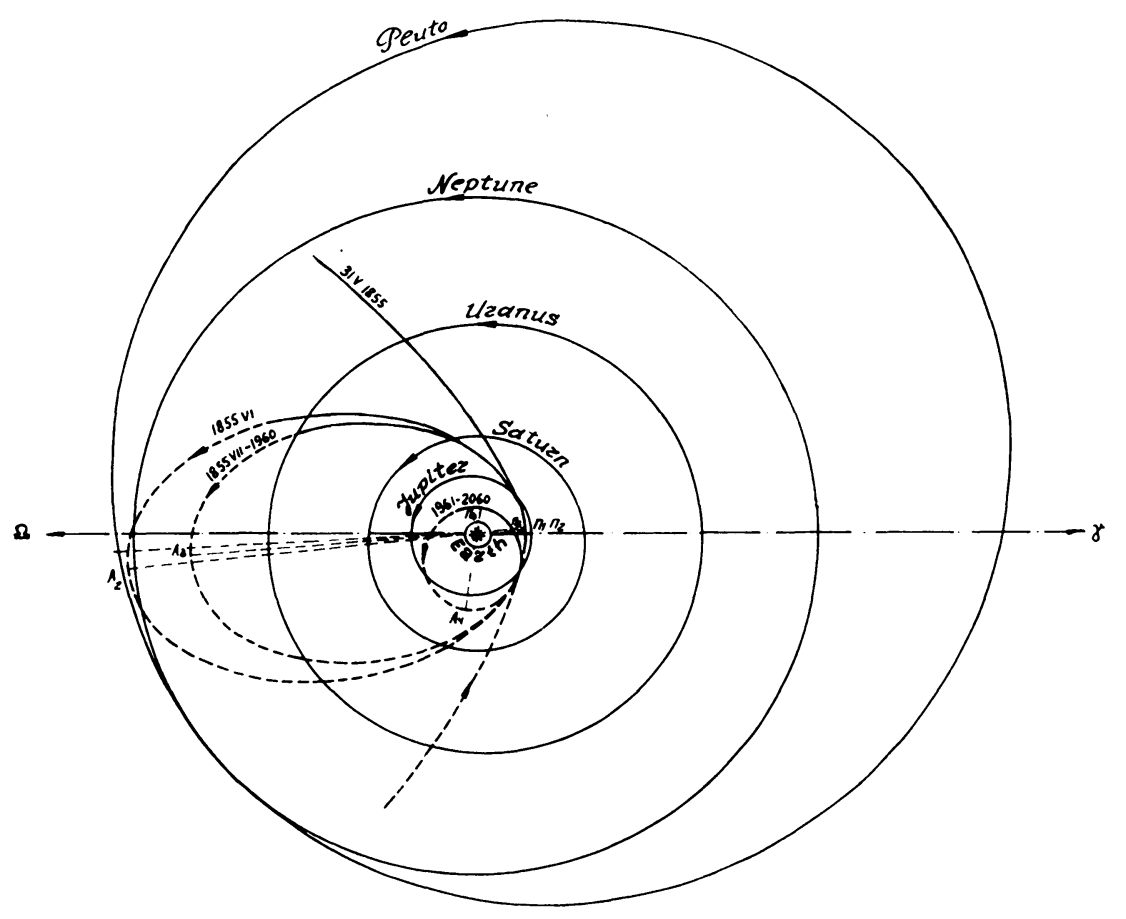

Fig. 10. The orbit of $P /$ Kearns-Kwee and the possible capture of this comet from a hyperbolic orbit.

starting orbit was not a definitive one we have been able to demonstrate the possibility, in principle, of Jupiter's capturing a comet from a hyperbolic orbit whose perihelion is near Jupiter's orbit. Our calculations have also shown a possible mechanism for this capture, first into an ellipse with its aphelion on the outskirts of the planetary system, and then into the Jupiter family.

\section{Fictitious Comets in the Spheres of Action of Uranus and Neptune}

We shall now discuss fictitious comets of the Uranus and Neptune families and possible transformations of their orbits in the spheres of action of those planets. Although we have studied many suitable fictitious objects we shall restrict ourselves here to only five: U-1 and U-2 in the Uranus family and $\mathrm{N}-1, \mathrm{~N}-2$, and $\mathrm{N}-3$ in the Neptune family.

Figure 11 illustrates the evolution of U-1 during the 10 -yr interval spent by that 
comet in the sphere of action of Uranus (1713-1723). The comet passed very deeply into the sphere (the radius of which is $0.346 \mathrm{AU}$ ) and in 1719 attained its minimum distance $\Delta_{\min }=0.0104 \mathrm{AU}$. The ascending node first regressed slowly, and then, in the vicinity of Uranus, it changed its direction of motion and rapidly advanced $167^{\circ}$, after which slow regression returned. The line of apsides turned through an angle of

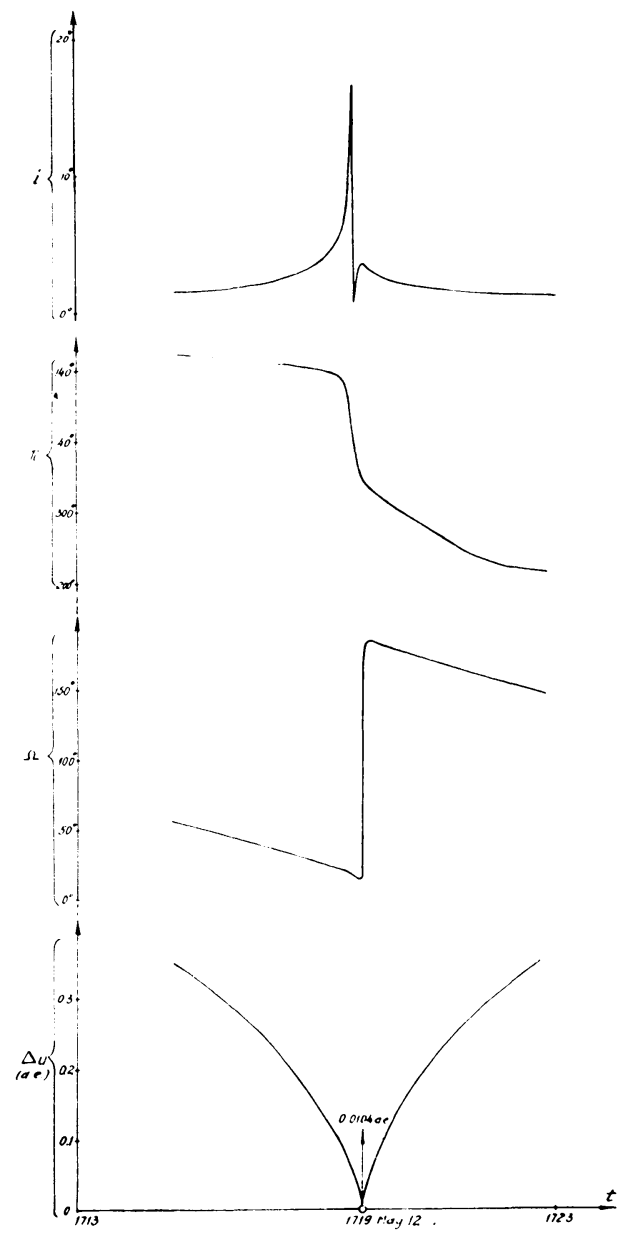

Fig. 11a.

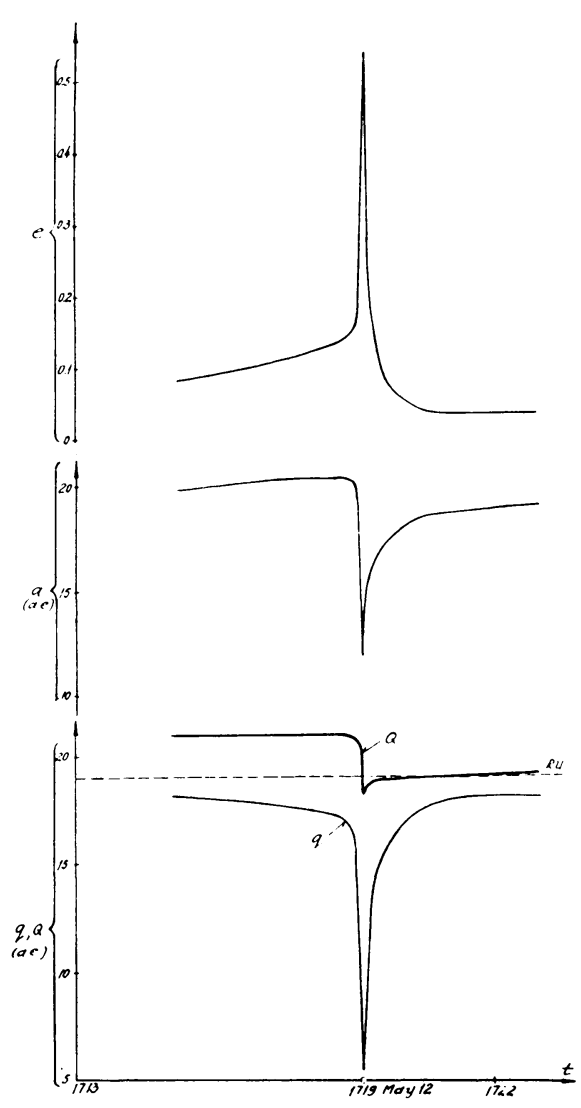

Fig. $11 b$.

Fig. 11a-b. Changes in the orbital elements of comet U-1 in Uranus' sphere of action.

$320^{\circ}$; the orbital inclination initially increased very slowly from 1.5 to 2.5 , then rose rapidly to $16: 3$, sharply decreased down to $0: 65$, rose to $3: 6$ and then slowly began to decrease down to $1: 2$, close to its initial value. On entering the sphere of activity the comet's orbit was nearly circular (semimajor axis $a=19.2 \mathrm{AU}$ and eccentricity $e=0.0614$ ) with its perihelion and aphelion just inside and just outside the orbit of 
Uranus. As the comet penetrated Uranus' activity sphere its orbit changed violently into an elongated ellipse with very small perihelion distance and decreasing semimajor axis $\left(a_{\min }=11.9 \mathrm{AU}\right)$; the aphelion moved inward, crossing the orbit of Uranus. As the comet receded from Uranus its orbit changed more or less back to its initial form.

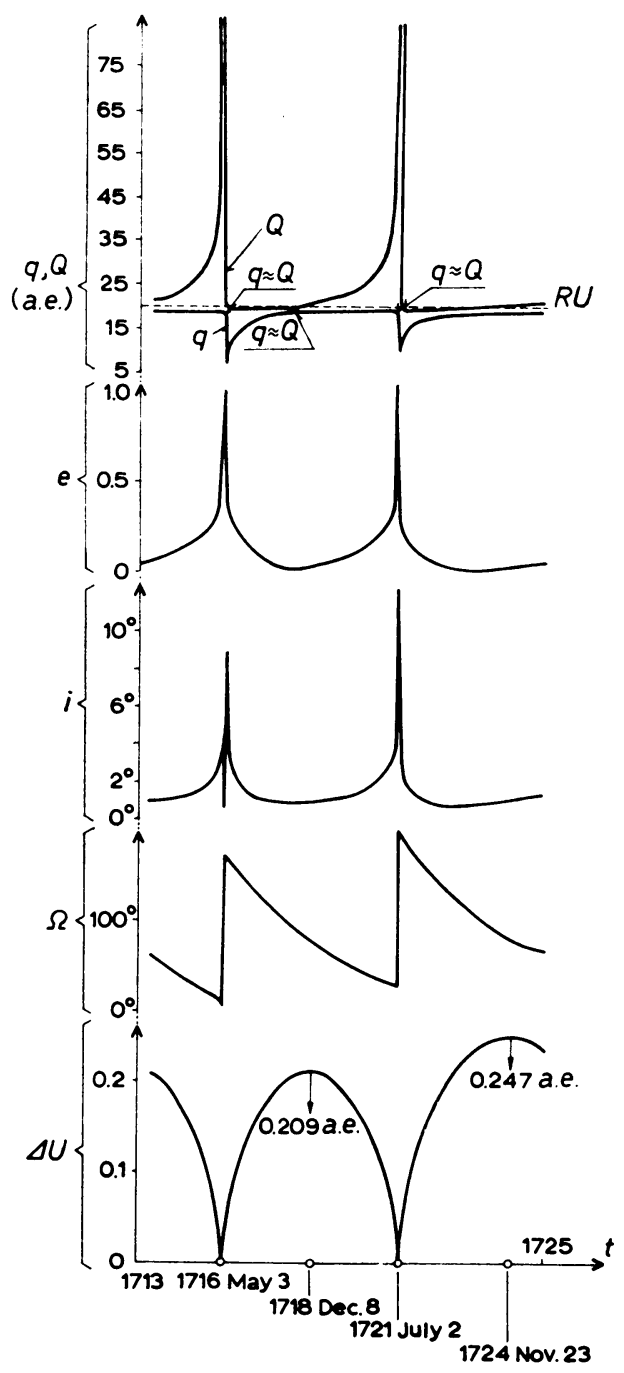

Fig. 12. Changes in the orbital elements of comet U-2.

The orbit of comet U-2 evolves in quite a different manner during its encounter with Uranus (1713-1725) (see Figure 12). After reaching its minimum distance from Uranus in $1716\left(\Delta_{\mathrm{min}}=0.0031 \mathrm{AU}\right)$ the comet receded, but it did not leave the sphere of action. Its distance increased to a maximum $\left(\Delta_{\max }=0.209 \mathrm{AU}\right)$, after which there was a second minimum $\left(\Delta_{\min }=0.0015 \mathrm{AU}\right)$ and another maximum $\left(\Delta_{\max }=0.247 \mathrm{AU}\right)$. 
During this time the cometary orbit changed twice from a low-eccentricity ellipse into a nearly parabolic orbit and back: on the first approach the eccentricity increased beyond 0.999 , while on the second the orbit became hyperbolic. The variations in perihelion and aphelion distances $(q, Q)$ are quite typical. When U-2 entered Uranus' sphere of action its perihelion was just inside the orbit of Uranus $(q=18.3 \mathrm{AU})$. At the time of the first minimum approach $q$ decreased suddenly to $7.30 \mathrm{AU}$; afterwards, the aphelion distance became approximately equal to the original $q$, and the perihelion distance slowly recovered to this value. Approximately the same process took place during the second minimum approach to Uranus.

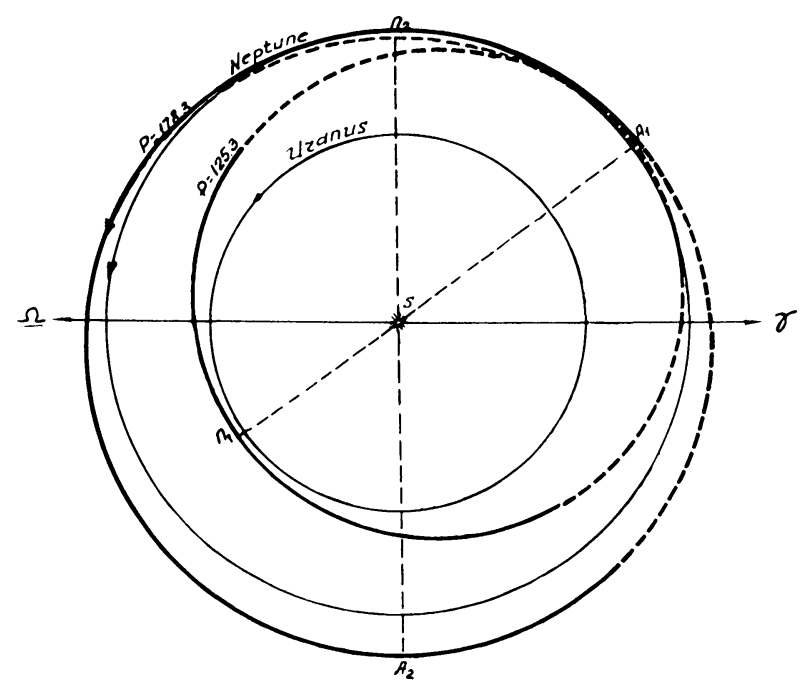

Fig. 13. The orbit of comet N-1.

Comet N-1 initially had an elliptical orbit with $a=25.04 \mathrm{AU}, e=0.199$, its perihelion $\Pi_{1}$ near the orbit of Uranus $(q=20.1 \mathrm{AU})$ and aphelion $A_{1}$ near that of Neptune $(Q=30.0 \mathrm{AU})$. It remained within Neptune's sphere of action (radius $0.580 \mathrm{AU}$ ) for about 13 yr (1708-1721), attaining $\Delta_{\min }=0.140$ AU. Figure 13 shows that $q$ increased by $9 \mathrm{AU}$, with the perihelion moving out to Neptune's orbit, and there was a slight increase in $Q$ also. The line of apsides rotated through $125^{\circ} .7$. On the other hand, the longitude of the ascending node (131:2) and inclination (1:77) were scarcely affected by the encounter.

Figures 14 and 15 illustrate the transformation of the orbit of comet N-2. Its close approach to Neptune in 1715 is unusual and interesting in that during an interval of little over a month there were three minimum approaches to Neptune $\left(\Delta_{\min }=0.0012\right.$ $\mathrm{AU}$, followed by $\Delta_{\max }=0.0063 \mathrm{AU}$, then $\Delta_{\min }=0.00093 \mathrm{AU}, \Delta_{\max }=0.0103 \mathrm{AU}$, and finally $\left.\Delta_{\min }=0.0028 \mathrm{AU}\right)$. During each encounter the orbit of $\mathrm{N}-2$ rapidly became retrograde and nearly parabolic and then direct and nearly parabolic. Figure 14 shows 


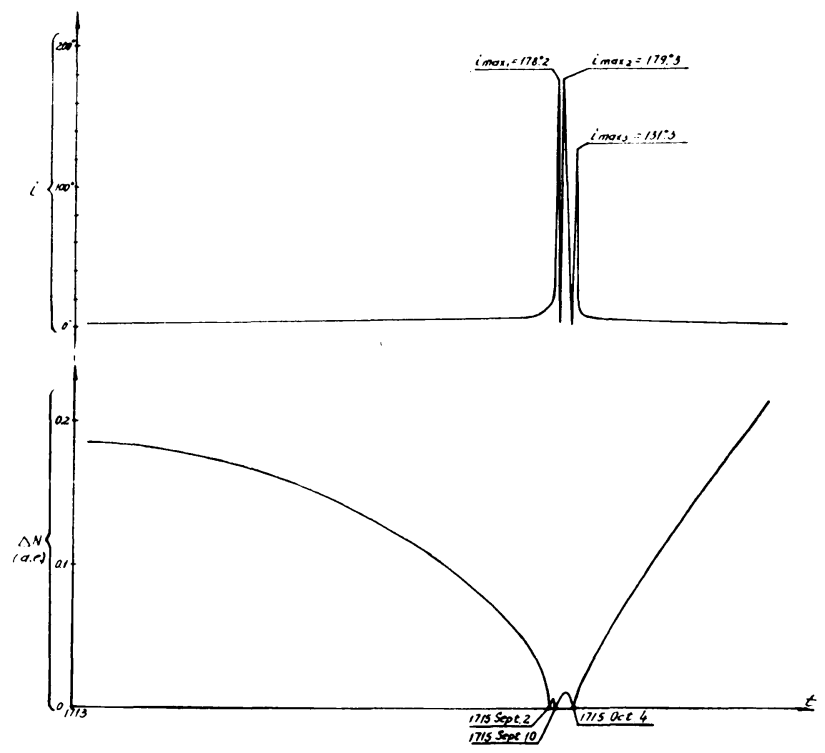

Fig. 14. The three approaches of comet N-2 to Neptune and the changes in orbital inclination.

that the inclination had three distinct maxima $\left(i_{\max _{1}}=178.2, i_{\max _{2}}=179.3, i_{\max _{3}}=\right.$ 131:3), corresponding to the three minimum approaches to Neptune. Figure 15 shows that after the comet's escape from Neptune's sphere of action the perihelion, initially

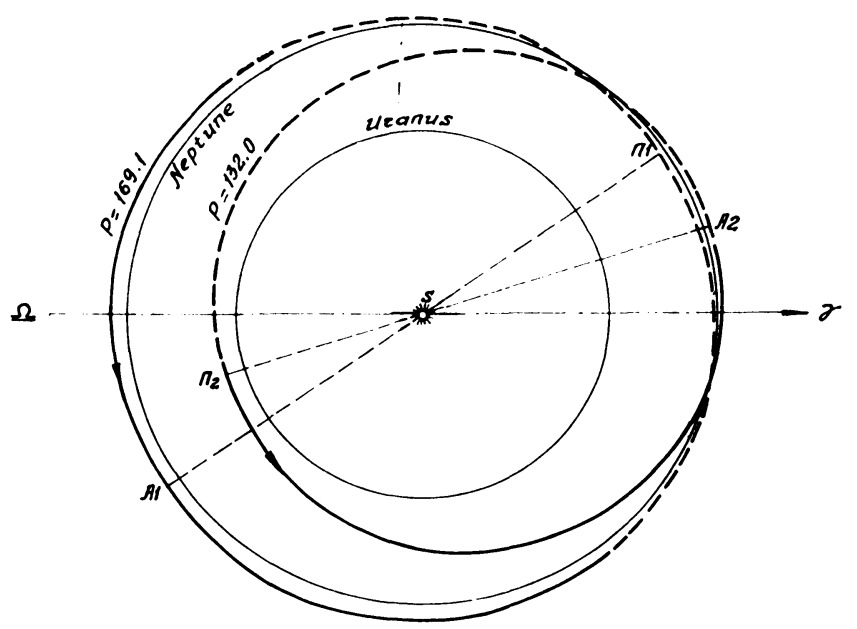

Fig. 15. The orbit of comet N-2.

near the orbit of Neptune $\left(\Pi_{1}\right)$, was moved to a point near the orbit of Uranus $\left(\Pi_{2}\right)$. The aphelion remained slightly outside the orbit of Neptune, but the line of apsides advanced through $161^{\circ}$. The eccentricity increased from 0.029 to 0.170 . The longitude of the ascending node advanced by $65^{\circ}$. 
Comet N-3 occupies a unique place among the fictitious comets of Neptune's family. Figure 16 illustrates the variation in the distance of $\mathrm{N}-3$ from Neptune during

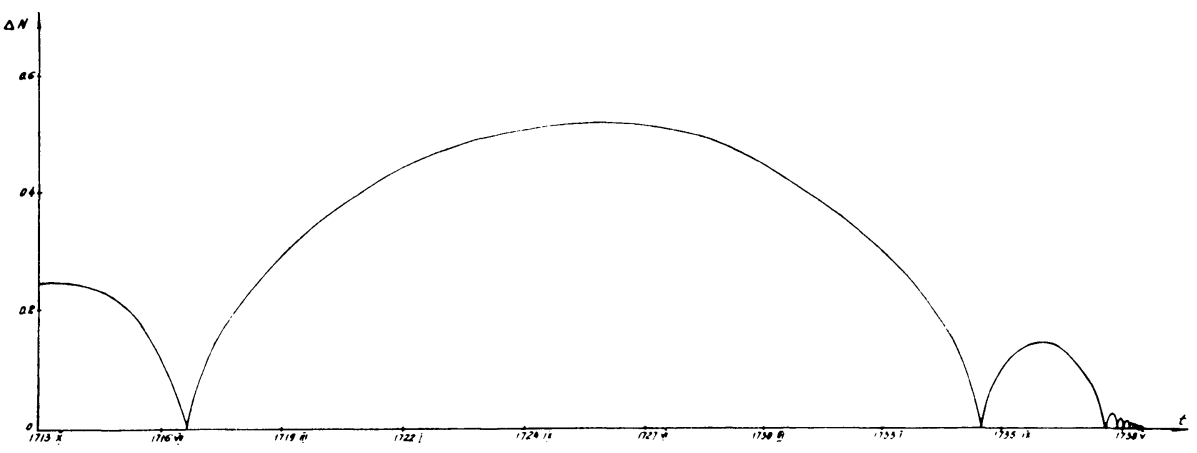

Fig. 16. The multiple approaches of comet N-3 to Neptune.

1713-1738; Table IV gives $\Delta_{\min }$ and $\Delta_{\max }$ and the corresponding dates $T_{\min }$ and $T_{\max }$ for the same interval. After the first minimum approach the comet travelled almost to

TABLE IV

Minimum and maximum distances of comet N-3 from Neptune

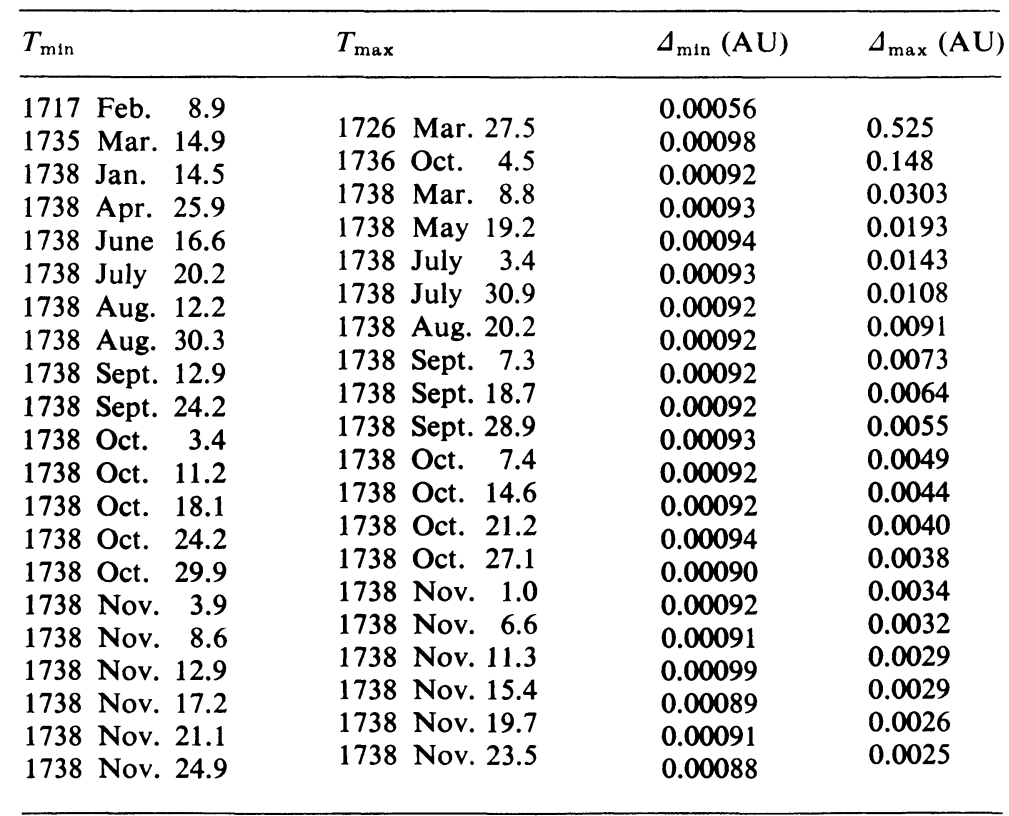

the periphery of Neptune's activity sphere before returning. Successive values of $\Delta_{\min }$ stabilized quickly near $0.0009 \mathrm{AU}$, while the values of $\Delta_{\max }$ decreased more 
slowly, the comet at the end of the integration run (at which point it seemed impractical to continue the calculation further, the step size having decreased to less than one hour) apparently having become a satellite of Neptune, the eccentricity of its neptunicentric orbit being about 0.5 and its revolution period less than four days.

Figures 17 and 18 illustrate the transformations in the various orbital elements (heliocentric) of N-3 during the first two minimum approaches to Jupiter. As happened with comet $\mathrm{N}-2$, very eccentric retrograde orbits are produced for a short time, the inclination reaching maximum values $i_{\max _{1}}=174.3$ and $i_{\max _{2}}=172^{\circ} .6$. As the differences between $\Delta_{\min }$ and $\Delta_{\max }$ diminish the heliocentric orbit of $\mathrm{N}-3$ asymptotically approaches that of Neptune itself.

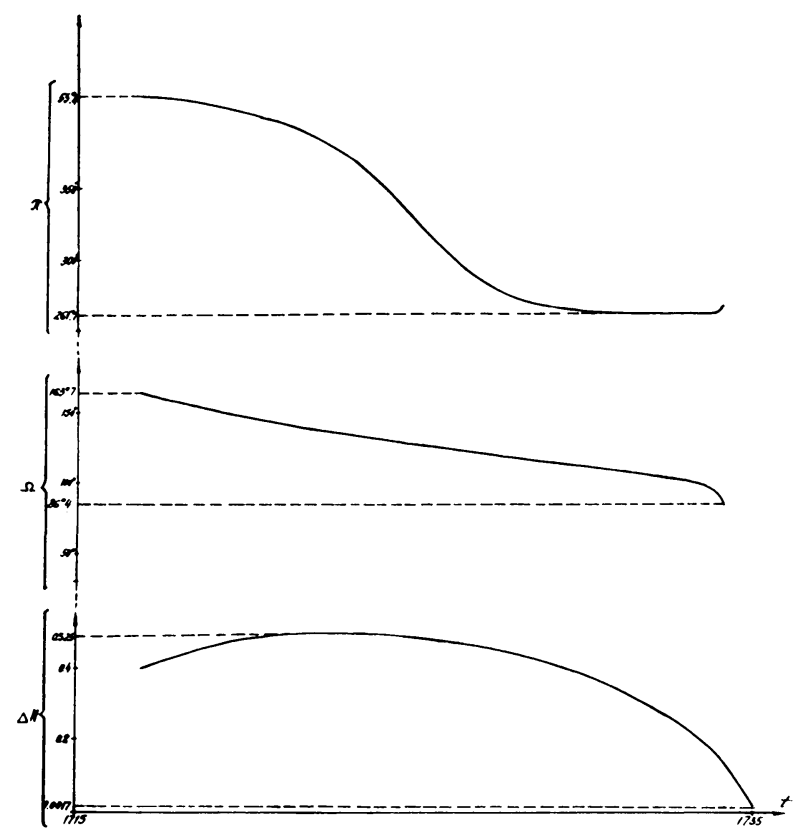

Fig. 17. Perturbations in the longitudes of the ascending node and perihelion of comet $\mathrm{N}-3$.

At the present stage of our work we have insufficient data for making any conclusions or generalities about the future of comets of the N-3 type; however, there is reason to believe that Neptune's sphere of action may contain a great number of comets that remain there indefinitely, accompanying Neptune in its motion round the Sun. Similar comets may also exist in the activity spheres of Jupiter, Saturn and Uranus.

\section{Discussion and Conclusions}

From the data obtained for 45 real and several dozen fictitious short-period comets we can draw some conclusions and give answers to some of the questions raised at the beginning of this paper. 
(1) The giant planets, Jupiter to Neptune, having large masses and extensive spheres of action, are powerful transformers of cometary orbits and appreciably affect their evolution; they change the spatial orientation of orbital planes and lines of apsides, vary the shapes and dimensions of the orbits, transfer comets from one planetary family to another and, in exceptional cases, remove them beyond the limits of the planetary system or capture them from hyperbolic orbits.

(2) Perturbations by the giant planets frequently create conditions favourable to the discovery of comets and on other occasions render comets inaccessible to observation.

(3) Scientific hypotheses on the origin of comets date back to Laplace and Lagrange. Laplace suggested that comets were of interstellar origin; Lagrange, on the other hand, supposed that they originated by eruption from the major planets, especially Jupiter. Each hypothesis has its adherents. Vsekhsvyatskij $(1930,1933,1955,1967,1969,1972)$ is a prominent supporter of Lagrange's ideas and has extensively developed the

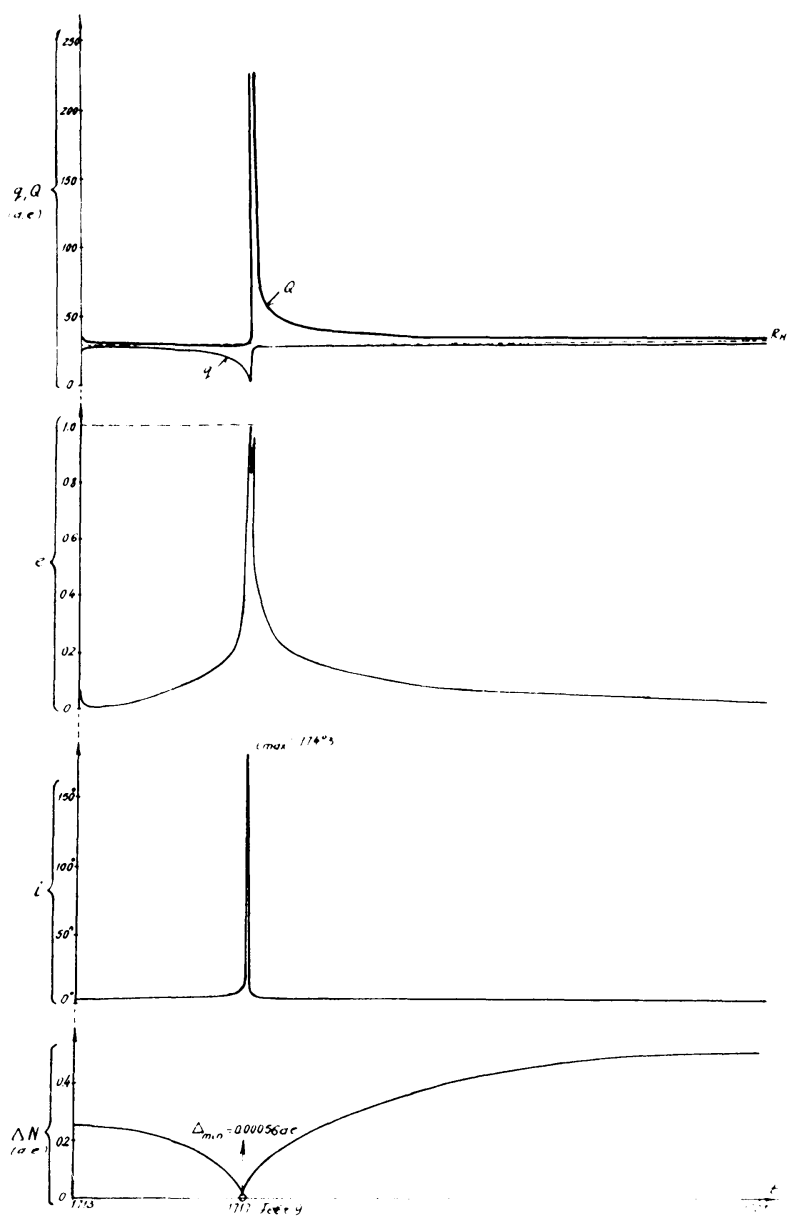

Fig. $18 \mathrm{a}$. 


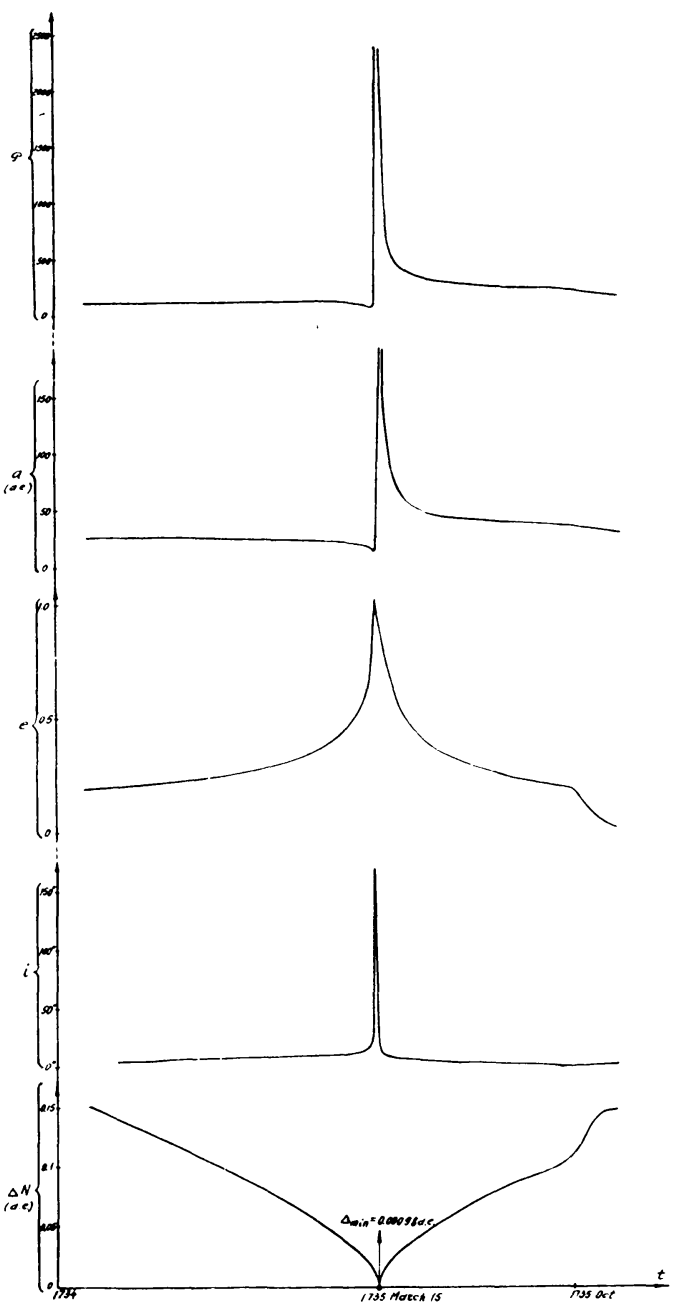

Fig. 18b.

Fig. 18a-b. Changes in the orbital elements of comet N-3.

'eruption theory'. Shtejns $(1960,1961,1962,1964,1972)$, Chebotarev (1972), as well as Belyaev, the present author and others prefer the 'capture theory', and we shall therefore discuss some of the conclusions of this theory in both its classical and its modern form.

The capture hypothesis of Laplace was further developed by Tisserand (1889), Schulhof (1891), Newton $(1878,1893)$, Callandreau (1892) and others. The classical studies were concerned with the transfer of a comet from interstellar space into the planetary system, i.e., transformation in Jupiter's activity sphere of an original parabolic orbit into an elliptical orbit with direct motion and short revolution period. In the 
initial studies the orbit of Jupiter was considered to be circular; in the sphere of Jupiter's action the jovicentric motion of the comet was taken to be Keplerian, perturbations by the Sun and other planets being ignored; outside that sphere perturbations (even those by Jupiter) were neglected. Under these idealized conditions Newton and Callandreau proved that, depending on the initial direction of motion, the orbit of the comet could be transformed by Jupiter into a short-period ellipse with direct motion, a retrograde ellipse, or a direct hyperbola. Everhart (1967, 1968, 1969, $1970,1972)$ has continued these investigations more extensively and with greater precision. But he did not make any substantially new conclusions concerning the capture of comets by the major planets.

It is well known that all the short-period comets of Jupiter's family possess elliptical orbits with direct motion. Supporters of the eruption theory maintain that the absence of observable comets with strongly hyperbolic orbits and retrograde short-period elliptical orbits presents unsurmountable difficulties for the capture theory. However, Schulhof stated that Jupiter would convert comets into retrograde elliptical orbits only rarely, and then at great jovicentric velocities. Callandreau (1892, p. 49) concluded that comets leaving Jupiter's sphere of action on hyperbolic orbits cannot be observed, either because their perihelion distances are too large, or because the comets do not pass through the perihelion points of these hyperbolic orbits.

Our investigations are in full agreement with those conclusions. Ellipses with retrograde motion and small perihelion distances do appear, but only very rarely, and only for a short time while the comets (e.g., N-2 and N-3) are well inside the activity spheres of the giant planets, i.e., at very great planetocentric velocities, as Schulhof surmised.

And as for the comets that leave Jupiter's sphere of action along hyperbolic orbits, our studies of numerous variants of the close approach of $\mathrm{P} /$ Lexell to Jupiter in 1779 fully confirm Callandreau's conclusion that the perihelia of such orbits do not exist in the range of observability.

Finally, we shall sketch - from the standpoint of celestial mechanics - the general picture of the evolution of cometary orbits, shedding new light on the capture theory in conjunction with the theory of diffusion of nearly parabolic comets and consideration of stellar perturbations.

Oort $(1950,1951)$ advanced a hypothesis according to which an extended 'cloud of comets' surrounds the solar system at a distance of about 150000 AU from the Sun. Fesenkov (1951) evaluated the diameter of the cloud as some tens of thousands of AU. Without discussing here the origin, stability, dimensions, and structure of this cloud, we shall regard the cloud as a collection of comets moving in nearly parabolic orbits and consider its formation the first stage in the evolution of cometary orbits. These problems are treated by Chebotarev (1964, 1966), Nezhinskij (1972), Antonov and Latyshev (1972), Yanovitskaya (1972), and others.

The second stage is determined by the stellar perturbations. This matter was first studied by Öpik (1932), then by Oort (1950) and Fesenkov (1951), and more recently by Shtejns (1962, 1964), Makover (1964), and Sekanina (1968). Shtejns established that stellar perturbations scarcely affect the semimajor axes of the cometary orbits, but they may very appreciably change the perihelion distances, even by hundreds of 
AU. Stellar perturbations thus may either reduce the perihelion distances into the region of the inner planetary system or remove the comets entirely. Makover has confirmed these conclusions with concrete examples.

The third stage is the diffusion of comets, the very gradual accumulation of small planetary perturbations in the reciprocal semimajor axes of the near-parabolic cometary orbits. This process is very slow, continuing for millions of years. The total changes in the semimajor axes may become quite considerable. Van Woerkom (1948) was the founder of the theory of cometary diffusion. The theory was later developed by Oort (1950, 1951), I.yttleton (1953), Shtejns and Riekstyn'sh (1960), Shtejns (1961), Shtejns and Sture (1962), Shtejns and Kronkalne (1964, 1968), Kendall (1961), and Whipple (1962).

Shtejns includes in his investigations all the nearly parabolic comets and those longperiod comets whose semimajor axes are not less than $40 \mathrm{AU}$. Using statistical methods he ascertains that diffusion produces a gradual reduction of the semimajor axes of a considerable fraction of the orbits of these comets without any change in perihelion distance. He also formulates the laws of cometary diffusion, taking into account the influence of an important astrophysical factor - the disintegration of cometary nuclei.

The first law of diffusion, discussed for the first time by Oort and then generalized by Shtejns, states that owing to diffusion comets having small semimajor axes also have small inclinations. The orbital planes of these comets gradually converge towards the plane of the ecliptic and consequently also to the mean plane of the orbits of the giant planets.

Shtejn's second law of diffusion states that cometary orbits having large perihelion distances also have small eccentricities.

The diffusion of comets thus reduces the orbital inclinations and leads to a gradual concentration of the perihelia of a multitude of invisible comets with nearly circular orbits on the periphery of the planetary system.

The fourth stage of the evolution is the capture of these invisible comets by the giant planets, and this has been the object of the investigations we have described here. Shtejns has studied by statistical methods most of the comets for which $a \geqslant 40 \mathrm{AU}$; we have investigated by the numerical methods of celestial mechanics comets with $a \leqslant 50$ AU. Together, therefore, the two investigations encompass all comets, and for those satisfying $40 \mathrm{AU} \leqslant a \leqslant 50 \mathrm{AU}$ two independent methods have been applied. Diffusion, taking place over millions of years, has produced comets moving in low-inclination, direct, nearly circular orbits at the edge of the planetary system.

Prolonged approaches of these comets to the giant planets (especially Neptune, with its large sphere of action) result in great transformations of their orbits. Favourable conditions are created for throwing these comets into the inner part of the solar system, where they can be observed. There also exist comets with low-inclination hyperbolic orbits and very elongated elliptical orbits whose perihelia are situated near the orbits of each of the giant planets. Capture is possible only when they penetrate the activity sphere of a planet near perihelion. Although a single catastrophic capture by Jupiter may occur, it is more probable that the capture of comets is a very compli- 
cated process, taking place over several millennia as the comets are thrown from one of the giant planets to another. There is no doubt that Jupiter plays the dominant role.

Undoubtedly, a considerable fraction of the comets leave the solar system under the influence of stellar or planetary perturbations. This is confirmed by the researches of Galibina (1958, 1963, 1964), Shtejns (1962, 1964), Brady (1965), Sekanina (1966a, 1966b), and by our investigations on $\mathrm{P} /$ Lexell.

The results discussed here are necessarily of a preliminary character. We fully realize that after constructing sufficiently precise numerical theories for all the shortperiod comets, combining all apparitions, and after repeated investigations of their orbital evolution with the highest precision, we may arrive at substantial modifications to the general picture of evolution. However, there is reason to believe that our researches on the orbital evolution of the short-period comets and on the role of the giant planets in this evolution will be of some importance, for they reveal for the first time a real mechanism for possible transformations of cometary orbits and elucidate the probable connection between long-period and short-period comets. These studies, conducted by the precise methods of celestial mechanics, characterize an important stage in the general evolution of cometary orbits within the boundaries of the planetary system; without taking these results into account it is impossible to create any scientific theory on the origin of comets.

\section{References}

Antonov, V. A. and Latyshev, I. N.: 1972, this Symposium, p. 341.

Belyaev, N. A.: 1966, Byull. Inst. Teor. Astron. 10, 696.

Belyaev, N. A.: 1967, Astron. Zh. 44, 461.

Brady, J. L.: 1965, Astron. J. 70, 279.

Callandreau, O.: 1892, Ann. Obs. Paris Mem. 20, B1.

Chebotarev, G. A.: 1964, Astron. Zh. 41, 983.

Chebotarev, G. A.: 1966, Astron. Zh. 43, 435.

Chebotarev, G. A.: 1972, this Symposium, p. 1.

Dinwoodie, C.: 1960, Quart. J. Roy. Astron. Soc. 2, 232.

Dubyago, A. D.: 1950, Trudy Astron. Obs. Kazan No. 31.

Dubyago, A. D.: 1956, Byull. Astron. Obs. V.P. Engel'gardta No. 32.

Everhart, E.: 1967, Astron. J. 72, 716.

Everhart, E.: 1968, Astron. J. 73, 1039.

Everhart, E.: 1969, Astron. J, 74, 735.

Everhart, E.: 1970, Astron. J. 75, 258.

Everhart, E.: 1972, this Symposium, p. 360.

Fesenkov, V. G.: 1951, Astron. Zh. $28,98$.

Galibina, I. V.: 1958, Byull. Inst. Teor. Astron. 6, 630.

Galibina, I. V.: 1963, Byull. Inst. Teor. Astron. 9, 46.

Galibina, I. V.: 1964, Byull. Inst. Teor. Astron. 9, 465.

Herget, P. and Marsden, B. G.: 1961, Quart. J. Roy. Astron. Soc. 2, 158.

Kamieński, M.: 1959, Acta Astron. 9, 53.

Kamieński, M.: 1961, Acta Astron. 11, 33.

Kazimirchak-Polonskaya, E. I.: 1967a, Trudy Inst. Teor. Astron. 12, 63, 86.

Kazimirchak-Polonskaya, E. I.: 1967b, Astron. Zh. 44, 439.

Kazimirchak-Polonskaya, E. I.: 1968, Astronomie 82, 217, 323, 432.

Kazimirchak-Polonskaya, E. I.: 1972, this Symposium, p. 95.

Kendall, D. G.: 1961, Proc. Fourth Berkeley Symposium on Math. Stat. and Probab. 3, 99, 121.

Leverrier, U. J. J.: 1848, Compt. Rend. Acad. Sci. Paris 26, 468. 
Leverrier, U. J. J.: 1857, Ann. Obs. Paris Mem. 3, 203.

Lexell, A. I.: 1777, Acta Acad. Sci. Petropol. 1, 332; 2, 328; Mem. Acad. Roy. Sci. Pruss. 638.

Lexell, A. I.: 1778, Acta Acad. Sci. Petropol. 1, 317; Acad. Sci. Petersb. 1.

Lexell, A. I.: 1781, Acta Acad. Sci. Petropol. 2, 351.

Lyttleton, R. A.: 1953, The Comets and their Origin, Cambridge.

Makover, S. G.: 1964, Byull. Inst. Teor. Astron. 9, 525.

Marsden, B. G.: 1964, IAU Circ. No. 1857.

Marsden, B. G. and Aksnes, K.: 1967, Astron. J. 72, 952.

Newton, H. A.: 1878, Am. J. Sci. Arts, New Haven, Conn. Ser. 316 (116), 165.

Newton, H. A.: 1893, Mem. Natl. Acad. Sci. Washington 6, 7.

Nezhinskij, E. M.: 1972, this Symposium, p. 335.

Oort, J. H.: 1950, Bull. Astron. Inst. Neth. 11, 91.

Oort, J. H.: 1951, Observatory 71, 129.

Öpik, E.: 1932, Proc. Am. Acad. Arts Sci. 67, 169; Harvard Repr. No. 79.

Schulhof, L.: 1891, Bull. Astron. 8, 147, 191, 225.

Sekanina, Z.: 1966a, Acta Univ. Carol. Math. Phys. $2,3$.

Sekanina, Z.: 1966b, Bull. Astron. Inst. Czech. 18, 1.

Sekanina, Z.: 1968, Bull. Astron. Inst. Czech. 19, 223, 291.

Shtejns, K. A.: 1960, Uch. Zap. Latv. Gos. Univ. 38, 69.

Shtejns, K. A.: 1961, Astron. Zh. 38, 107, 304.

Shtejns, K. A.: 1962, Astron. Zh. 39, 915.

Shtejns, K. A.: 1964, Uch. Zap. Latv. Gos. Univ. 68, 39.

Shtejns, K. A.: 1972, this Symposium, p. 347.

Shtejns, K. A. and Kronkalne, S.: 1964, Acta Astron. 14, 311.

Shtejns, K. A. and Kronkalne, S.: 1968, Izv. Akad. Nauk Latv. SSR 9, 59.

Shtejns, K. A. and Riekstyn'sh, E. I.: 1960, Astron. Zh. 37, 1061.

Shtejns, K. A. and Sture, S. J.: 1962, Astron. Zh. 39, 506.

Sitarski, G.: 1968, Postępy Astron. 16, 159.

Tisserand, F.: 1889, Bull. Astron. 6, 241, 289.

van Woerkom, A. J.: 1948, Bull. Astron. Inst. Neth. 10, 445.

Vsekhsvyatskij, S. K.: 1930, Monthly Notices Roy. Astron. Soc. 90, 706; Astron. Nachr. 240, 273.

Vsekhsvyatskij, S. K.: 1933, Astron. Zh. 10, 18.

Vsekhsvyatskij, S. K.: 1955, Astron. Zh. 32, 432.

Vsekhsvyatskij, S. K.: 1967, Priroda i Proiskhozhdenie Komet i Meteornogo Veshchestva, Prosveshchenie, Moscow.

Vsekhsvyatskij, S. K.: 1969, Problemy Sovremennoj Kosmogonii, Moscow, p. 240.

Vsekhsvyatskij, S. K.: 1972, this Symposium, p. 413.

Whipple, F. L.: 1962, Astron. J. 67, 1.

Wiśniewski, W.: 1960, IAU Circ. No. 1729.

Yanovitskaya, G. T.: 1972, this Symposium, p. 346.

\section{Discussion}

S. K. Vsekhsvyatskij: Your statement on the unobservability of hyperbolic comets with perihelia on Jupiter's orbit is contradicted by the fact that other comets (P/Schwassmann-Wachmann 1 for instance) are observed far beyond a distance of $5 \mathrm{AU}$ from the Sun.

E. I. Kazimirchak-Polonskaya: Comets more than $5 \mathrm{AU}$ from the Sun are invariably faint and observable only because their orbits have been determined from observations made at smaller heliocentric distances, and we thus know where to find them. P/Schwassmann-Wachmann 1 was discovered only because of its unusual surges in brightness.

$V$. V. Fedynskij: May one consider that the 'pulsating ellipses', such as you noted for $\mathrm{P} / \mathrm{Wolf}$, is a typical mechanism in the evolution of the orbits of short-period comets?

E. I. Kazimirchak-Polonskaya: Yes. Analogous fluctuations were found by Belyaev in the case of $\mathrm{P} /$ Schwassmann-Wachmann 2 and by myself for $\mathrm{P} /$ Wolf-Harrington and other comets. 